\title{
FREE CONVECTION FLOW IN A VERTICAL THIN CYLINDER OF FINITE HEIGHT WITH POWER LAW FLUIDS
}

\author{
NGUYEN VAN QUE \\ Institute of Mechanics, NCNST of Vietnam
}

\section{INTRODUCTION}

In [1] free convection flow in a vertical channel of finite height and thickness with power law fluid is investigated.

In this paper we consider free convection flow in a vertical thin cylinder of finite height with given external temperature (see Fig. 1). The problem is solved by a finite difference scheme. The calculation result when the height is much bigger than the diameter is compared with asymptotic one. A condition of neglecting the thickness is shown.

\section{BASIC EQUATIONS AND ESTABLISHING THE PROBLEM}

According to the boundary layer theory and Bushinhesc approximation, in cylindrical coordinates the problem is governed by following equations in dimensionless form (see $[2,3])$.

Continuity equation:

$$
\frac{\partial \bar{r} \bar{v}_{r}}{\partial \bar{r}}+\frac{\partial \bar{r} \bar{v}_{z}}{\partial \bar{z}}=0
$$

Momentum equation:

$$
\bar{v}_{r} \frac{\partial \bar{v}_{r}}{\partial \bar{r}}+\bar{v}_{z} \frac{\partial \bar{v}_{z}}{\partial \bar{z}}=-\frac{d \bar{p}^{\prime}}{d \bar{z}}+\frac{1}{\bar{r}} \frac{\partial}{\partial \bar{r}}\left(\bar{r}_{\eta} \bar{v}_{z, r}\right)+\bar{T} G_{r g}
$$

Energy equation:

$$
\begin{gathered}
\bar{v}_{r} \frac{\partial \bar{T}}{\partial \bar{r}}+\bar{v}_{z} \frac{\partial \bar{T}}{\partial \bar{z}}=\frac{1}{\bar{r}} \frac{\partial}{\bar{r}}\left(\bar{r} \frac{\partial \bar{T}}{\partial x}\right) \cdot P_{r g}^{-1} ; \\
\frac{1}{\bar{r}} \frac{\partial}{\partial \bar{r}}\left(\bar{r} \frac{\partial \bar{T}_{1}}{\partial \bar{r}}\right)+(D / H)^{2} \frac{\partial^{2} \bar{T}_{1}}{\partial \bar{z}^{2}}=0 ; \quad \frac{1}{2} \leq r \leq \frac{1}{2}+\bar{\delta}
\end{gathered}
$$

where

$$
\begin{gathered}
\bar{z}=\frac{z}{H}, \quad \bar{r}=\frac{r}{D}, \quad \bar{\delta}=\frac{\delta}{D}, \quad \bar{v}_{z}=\frac{v_{z} D}{H u^{*}}, \quad \bar{v}_{r}=\frac{v_{r}}{u^{*}}, \\
\bar{T}=\frac{T-T_{\infty}}{T_{w}-T_{\infty}}, \quad \bar{T}_{1}=\frac{T_{1}-T_{\infty}}{T_{w}-T_{\infty}}, \quad \bar{p}^{\prime}=\frac{p^{\prime} D^{2}}{\rho u^{*} H}, \quad \eta=\left|\bar{v}_{z, r}\right|^{n-1} \\
P_{r g}=C_{p} \rho u^{*} D \lambda^{-1}, \quad G_{r g}=g \beta\left(T_{w}-T_{\infty}\right) u_{*}^{-2} H^{-1} D^{2}, \quad u^{*}=\nu_{k}^{\frac{1}{2-n}} D^{\frac{1-3 \pi}{3-n}} H^{\frac{n-1}{2-n}} .
\end{gathered}
$$


$\eta$ - apparent viscosity, $D=2 R, \delta$ - the wall thickness, $T_{\infty}$ - temperature of surroundings, $T_{w}$ temperature at external wall, $T_{1}$ - the temperature inside the wall, $p^{\prime}=p(z)-p(0)+g \rho z, P_{r g}, G_{r g}$ - generalized Prantl and Grashof numbers, $\nu_{k}$ - kinematics viscosity, $\rho$-density, $C_{p}$ - specific heat coefficient, $\lambda$ - thermal conductivity, $g$ - acceleration of gravity, $\beta$ - thermal expansion coefficient.

Boundary conditions:

$$
\begin{aligned}
\bar{v}_{r}\left(\frac{1}{2}, \bar{z}\right) & =\bar{v}_{z}\left(\frac{1}{2}, \bar{z}\right)=0 \\
\bar{v}_{r}(0, \bar{z}) & =\frac{\partial \bar{v}_{z}}{\partial \bar{r}}(0, \bar{z})=\frac{\partial \bar{T}}{\partial \bar{r}}(0, \bar{z})=0 ; \\
\bar{T}_{1}\left(\frac{1}{2}+\bar{\delta}, \bar{z}\right) & =T_{w} ; \quad \bar{T}_{1}\left(\frac{1}{2}, \bar{z}\right)=\bar{T}\left(\frac{1}{2}, \bar{z}\right), \\
\lambda_{1} \frac{\partial \bar{T}_{1}}{\partial \bar{r}}\left(\frac{1}{2}, \bar{z}\right) & =\lambda \frac{\partial \bar{T}}{\partial \bar{r}}\left(\frac{1}{2}, \bar{z}\right) ; \\
p^{\prime}(0) & =\bar{v}_{r}(\bar{r}, 0)=\bar{T}(\bar{r}, 0)=0 ; \\
\bar{v}_{z}(\bar{r}, 0) & =v_{z 0} ; \quad \bar{p}^{\prime}(1)=0 .
\end{aligned}
$$

Because of the smallness of $\delta$ in comparison with $H ;\left(\frac{\delta}{H}\right) \ll 1$ the second term in (2.4) can be neglected. This leads to the following equation:

$$
\frac{\partial}{\bar{\partial} \bar{r}}\left(\bar{r} \frac{\partial \bar{T}_{1}}{\partial \bar{r}}\right)=0 \quad \frac{1}{2} \leq \bar{r} \leq \frac{1}{2}+\bar{\delta}
$$

In addition, from the continuity equation and condition $\bar{v}_{r}\left(\frac{1}{2}, \bar{z}\right)=0$ it follows:

$$
\int_{0}^{1 / 2} \bar{r} \bar{v}_{z} d \bar{r}=\text { const }=\frac{1}{8} v_{z 0}
$$

The unknowns of system $(2.1)-(2.7)$ are $\bar{v}_{r}(\bar{r}, \bar{z}), \bar{v}_{z}(\bar{r}, \bar{z}), \bar{T}(\bar{r}, \bar{z}), \bar{T}_{1}(\bar{r}, \bar{z}), \bar{p}^{\prime}(\bar{z}), v_{z 0}$.

Two quantities of particular interest are the average velocity along the channel $v_{z 0}$ and the total heat transfer from the wall $Q$, which is characterized by average Nusselt number $\bar{N}_{u_{D}}$

\section{NUMERICAL SOLUTIONS}

First, we can exclude $T_{1}$ by integrating (2.6) combining with (2.5), and we get following boundary condition for $\bar{T}$ at $\bar{r}=\frac{1}{2}$ :

$$
2 \psi\left(1-\bar{T}\left(\frac{1}{2}, \dot{z}\right)\right)=\frac{\partial \bar{T}}{\partial \bar{r}}\left(\frac{1}{2}, \bar{z}\right)
$$

where $\psi=\frac{\lambda_{1}}{\lambda \ln (1+2 \bar{\delta})}$.

After $T$ has found $T_{1}$ can be calculated as

$$
T_{1}=\frac{1-T\left(\frac{1}{2}, \bar{z}\right)}{\ln (1+2 \bar{\delta})} \ln \bar{r}+\frac{T\left(\frac{1}{2}, \bar{z}\right) \ln \left(\frac{1}{2}+\bar{\delta}\right)+\ln 2}{\ln (1+2 \bar{\delta})}
$$

(2.1) - (2.4), (2.7), (3.1) is a closed system for $\bar{v}_{r}(\bar{r}, \bar{z}), \bar{v}_{z}(\bar{r}, z), \bar{T}(\bar{r}, \bar{z}), \bar{p}^{\prime}(\bar{z}), v_{z_{0}}$. 
We solve this system by a finite difference method. The finite difference equation (see Fig. 2) (drop signs - for convenience)

$$
\begin{aligned}
& \frac{\left(r^{s+1} v_{r}\right)_{k+1}^{J+1}-\left(r \dot{v}^{s+1} r\right)_{k}^{J+1}}{\Delta r}+\frac{\left(r^{s} v_{z}\right)_{k+1}^{J+1}+\left(r^{s+1}{ }_{z}\right)_{k}^{J+1}-\left(r v_{z}\right)_{k+1}^{J}-\left(r v_{z}\right)_{k}^{J}}{2 \Delta z}=0
\end{aligned}
$$

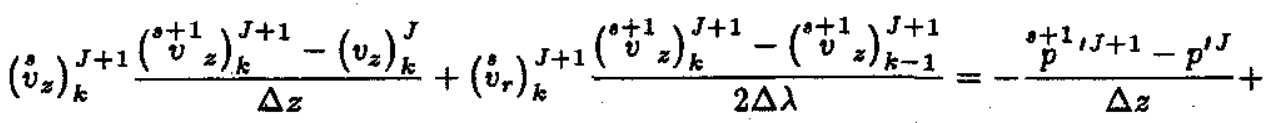

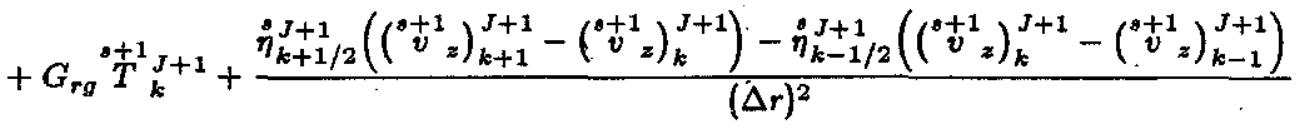

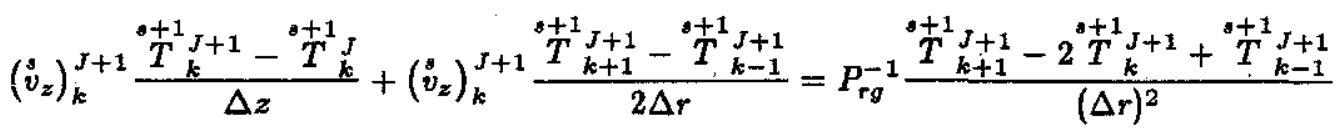

where $\eta_{k+1 / 2}, \eta_{k-1 / 2}$ is taken equal to $\left|\frac{u_{k+1}-u_{k}}{\Delta r}\right|^{n-1} ;\left|\frac{u_{k}-u_{k-1}}{\Delta r}\right|^{n-1}$.

This is a non-linear system. The truncation errors is of $O\left(\Delta z, \Delta r^{2}\right)$. The Von Neuman stability condition is satisfied unconditionally.

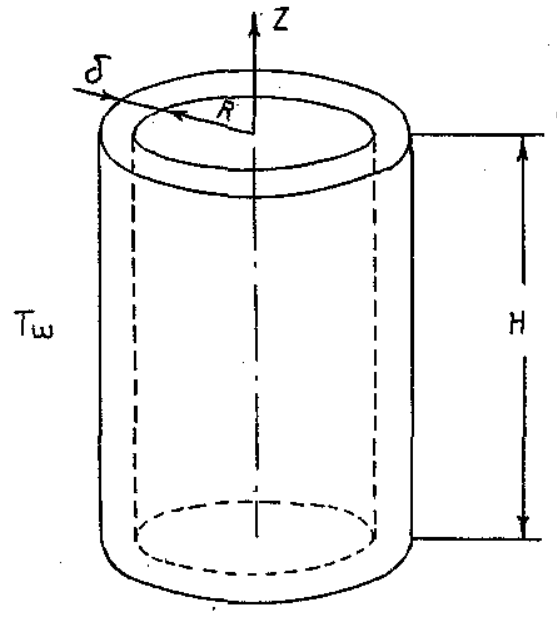

Fig. 1

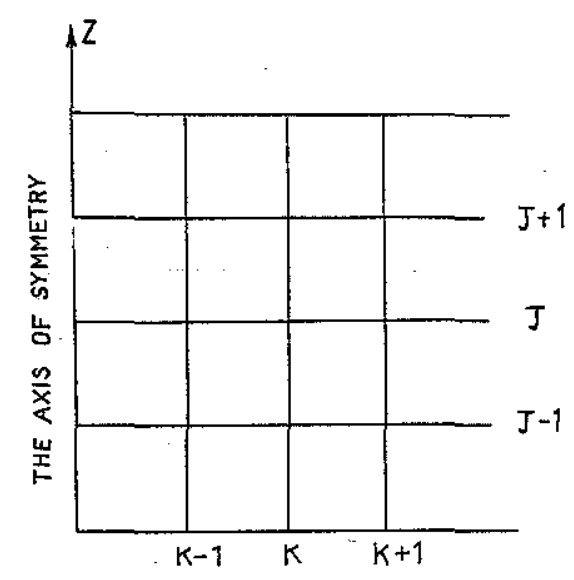

Fig. 2

We solve this system by iterating on index s: Let's assume that all quantities at $J$ - row and quantities with index $s$ at $J+1$ - row are known. From (3.4) using the Thomas algorithm we can obtain ' $T^{+1}{ }^{J+1}$. Introducing into (3.3) gives (drop index $s+1$ and $J+1$ at $v_{z}$ and $p^{\prime}$ for convenience).

$$
\begin{gathered}
A_{k}\left(v_{z}\right)_{k-1}+B_{k}\left(v_{z}\right)_{k}+C_{k}\left(v_{z}\right)_{k+1}+p^{\prime}=D_{k} ; \quad k=\overline{2, N-1} \\
\left(v_{z}\right)_{1}=\left(v_{z}\right)_{2} ; \quad\left(v_{z}\right)_{N}=0 \\
\int_{0}^{1 / 2} r v_{z} d r=\frac{1}{8} v_{z_{0}}
\end{gathered}
$$


(3.5), (3.6) are $(N+1)$ equations for $(N+1)$ unknowns $p^{\prime},\left(v_{z}\right)_{1},\left(v_{z}\right)_{2}, \ldots,\left(v_{z}\right)_{N}$. We solve this system as follows:

Let $p_{1}, p_{2} ; p_{1} \neq p_{2}$ - two arbitrary values. Using the Thomas algorithm we can find two solution $v_{z}^{(1)}, v_{z}^{(2)}$ :

$$
\begin{aligned}
& v_{z}^{(1)}=\left(\left(v_{z}\right)_{1}^{(1)},\left(v_{z}\right)_{2}^{(1)}, \ldots,\left(v_{z}\right)_{N}^{(1)}\right) \\
& v_{z}^{(2)}=\left(\left(v_{z}\right)_{1}^{(2)},\left(v_{z}\right)_{2}^{(2)}, \ldots,\left(v_{z}\right)_{N}^{(2)}\right)
\end{aligned}
$$

of system (3.5). Because of the linearity $\alpha p_{1}+(1-\alpha) p_{2}, \alpha v_{z}^{(1)}+(1-\alpha) v_{z}^{(2)} ; \forall \alpha$ are solutions of (3.5), too. Substitution into (3.6) gives:

$$
\alpha=\frac{\frac{1}{8} v_{z_{0}}-\int r v_{z}^{(2)} d r}{\int_{0}^{1 / 2} r\left(v_{z}^{(1)}-v_{z}^{(2)}\right) d r} .
$$

After ${ }^{s+1} v_{z}^{J+1}$ has found, introducing into (3.2) we can find ${ }^{s+1} v_{r}^{J+1}$ and so on, until the variables with index $s+1$ coincide with the variables with index 3 . The initial values are taken equal to values at $J$. At entrance $z=0$ a given (guessed) valued of $v_{z_{0}}$ was used as starting value. If the calculation up to $z=1$ yielded a value of $p^{\prime}(1)$ of zero then the correct value of $v_{z_{0}}$ had been used. If not the process was repeated until $p^{\prime}(1)$ was zero.

\section{DISCUSSION OF THE RESULTS}

a) Asymptotic solution

When $(H / D) \rightarrow \infty$ then far from the entrance the problem is one-dimensional and we can find the solution easily

$$
\begin{aligned}
T & =1 \\
v_{z} & =\frac{n}{n+1} G_{r g}^{1 / n}\left[(1 / 2)^{1+\frac{1}{n}}-r^{1+\frac{1}{n}}\right]
\end{aligned}
$$

It yields

$$
v_{z_{0}}=\frac{n}{3 n+1} G_{r g}^{1 / n}\left(\frac{1}{2}\right)^{1+\frac{1}{n}}
$$

If $h$ stands for average heat transfer to the liquid coefficient then

$$
\begin{aligned}
h & =\frac{\lambda}{D} \cdot \frac{1}{4} \frac{n}{3 n+1} P_{r g} \cdot G_{r g}^{1 / n}\left(\frac{1}{2}\right)^{1+\frac{1}{n}} \\
\bar{N}_{u_{D}} & =\frac{h D}{\lambda}=\frac{1}{4} \frac{n}{3 n+1} P_{r g} \cdot G_{r g}^{1 / n}\left(\frac{1}{2}\right)^{1+\frac{1}{n}}
\end{aligned}
$$

For comparison we take $P_{r g}=100 ; G_{r g}=4,79 \cdot 10^{-2}, n=0,66 ; \frac{\lambda_{1}}{\lambda}=4, \bar{\delta}=\frac{1}{8}$

(4.2), (4.3) give

$$
\begin{aligned}
v_{z_{0}} & =3.87 \cdot 10^{-4} \\
\bar{N}_{u_{D}} & =9.68 \cdot 10^{-3}
\end{aligned}
$$


Numerical solution are

$$
\begin{aligned}
v_{z_{0}} & =3.74 \cdot 10^{-4} \\
\bar{N}_{u_{D}} & =9.57 \cdot 10^{-3} \quad\left(Q=3.59 \cdot 10^{-2} W\right)
\end{aligned}
$$

The differences are smaller $3.5 \%$.

b) Numerical example

The fluid under consideration is a 1000 wppm solution of water and CMC (carboxy methyl cellulose). The input data are as follows (see [2])

$$
\begin{array}{ll}
T_{w}=25^{\circ} \mathrm{C} & T_{\infty}=15^{\circ} \mathrm{C} \\
D=2 \mathrm{~cm} & H=20 \mathrm{~cm} \\
\rho=1000 \mathrm{~kg} / \mathrm{m}^{3} & C_{p}=4.18 \cdot 10^{3} \mathrm{~J} / \mathrm{kgK} \\
\lambda=0.597 \mathrm{~W} / \mathrm{mK} K & \nu_{k}=7.35 \cdot 10^{-8} \mathrm{~m}^{2} / \mathrm{s}^{2-n} \\
\beta=1.8 \cdot 10^{-4} 1 / K & n=0.66, \quad \delta=\frac{D}{8} .
\end{array}
$$

The calculation results are $v_{z_{0}}=3.26 \cdot 10^{-2}($ that is $0.103 \mathrm{~cm} / \mathrm{s}) ; \bar{N}_{u_{D}}=1.94(Q=7.26 \mathrm{~W})$ for the case of $\psi=\frac{\lambda_{1}}{\lambda \ln (1+2 \bar{\delta})}=17.9$. The distributions of $T, v_{r}, v_{z}$ are shown in figures $3,4,5$. Comparing with the case of wall thickness ignored [3] we see that the wall thickness reduces the convection as well as the heat transfer. This influence is characterized by parameter $\psi$ solely.

The bigger $\psi$ is the smaller the influence is. Calculation shows that when $\psi \geq 100$ the differences caused by wall thickness is smaller $2 \%$ so we can neglect it

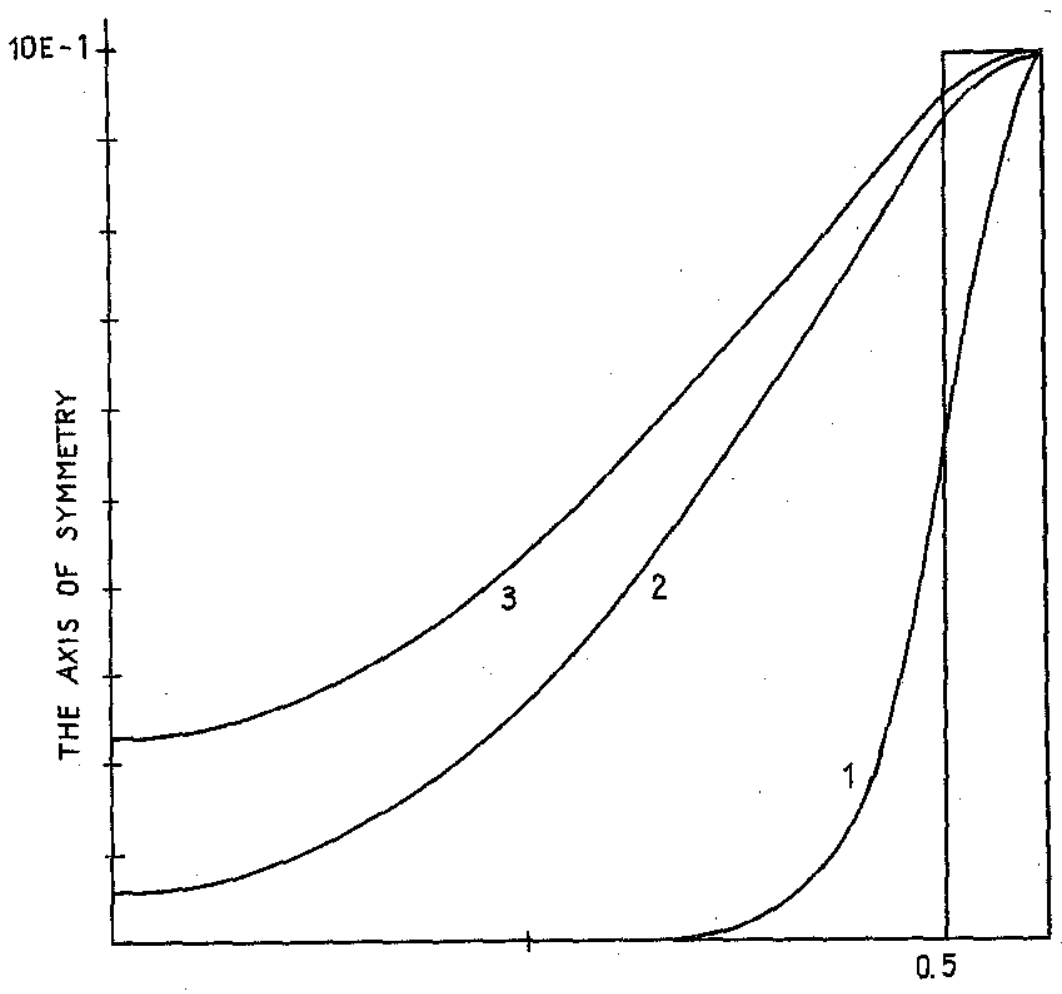

Fig. 9. Dimensioniess temperature distribution

1. at $z=2.5 \cdot 10^{-5} \mathrm{H}$, 2. at $z=0.5 \mathrm{H}$, 3. at $z=H$ 


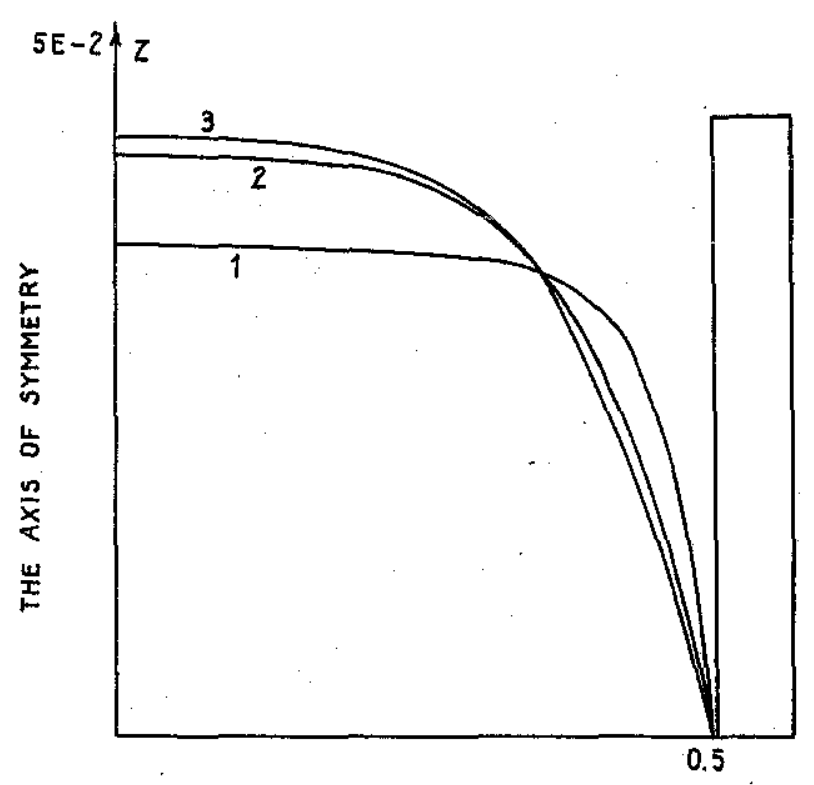

Fig. 4. Dimensionless component $v_{z}$ distribution 1. at $z=2.5 \cdot 10^{-5} H$, 2. at $z=0.5 H$, 3. at $z=H$

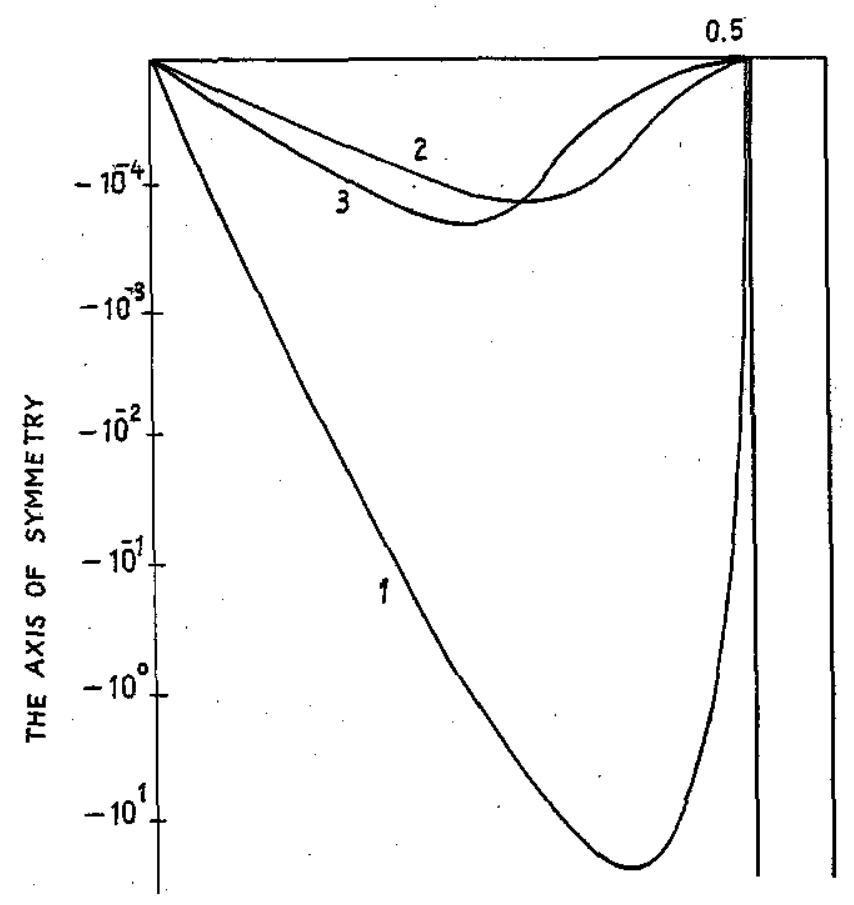

Fig. 5. Dimensionless component $v_{\text {r distribution }}$

1. at $z=2.5 \cdot 10^{-5} H$, 2. at $z=0.5 H$, 3. at $z=H$

Author is grateful to Dr of Sc. Prof. Ngo Huy Can, Dr of Sc. Vu Duy Quang, Ph. D. Pham Hung for many helpful suggestions.

This publication is completed with financial support from the National Basic Research Program in Natural sciences.

(xem tiếp trang 48) 